\title{
Quantum Baker Maps for Spiraling Chaotic Motion
}

\author{
Pedro R. del Santoro, Raúl O. Vallejos, and Alfredo M. Ozorio de Almeida \\ Centro Brasileiro de Pesquisas Físicas (CBPF), Rua Dr. Xavier Sigaud 150, 22290-180 Rio de Janeiro, Brazil
}

Received on 22 January, 2007

\begin{abstract}
We define a coupling of two baker maps through a $\pi / 2$ rotation both in position and in momentum. The classical trajectories thus exhibit spiraling, or loxodromic motion, which is only possible for conservative maps of at least two degrees of freedom. This loxodromic baker map is still hyperbolic, that is, fully chaotic. Quantization of this map follows on similar lines to other generalized baker maps. It is found that the eigenvalue spectrum for quantum loxodromic baker map is far removed from those of the canonical random matrix ensembles. An investigation of the symmetries of the loxodromic baker map reveals the cause of this deviation from the Bohigas-Giannoni-Schmit conjecture.
\end{abstract}

Keywords: Quantum chaos; Baker map; Spiraling motion

\section{INTRODUCTION}

The essential feature of classically chaotic motion is hyperbolicity, that is both stretching and squeezing, within a confined region of phase space. The simplest textbook examples, as far as (conservative) Hamiltonian systems are concerned, are two-dimensional $(2 D)$ maps, which may be pictured as Poincaré sections of motion in a $4 D$ phase space. However, hyperbolicity is in no way confined to lower dimensions. In particular, a conservative return map in four dimensions may exhibit spiralling, or loxodromic motion onto a fixed point. This would not be allowed in two dimensions since it would violate area conservation, but is compensated in four dimensions by outward spiralling in a complementary plane. The linearization of hyperbolic conservative maps of arbitrarity high dimension can be decomposed into products of simple hyperbolic motion in eigenplanes together with loxodromic motion in $4 D$-subspaces [1].

Hyperbolic motion is generally described by horseshoe maps in the qualitative theory of dynamical systems [2, 3]. The idea is to describe by symbolic dynamics the successive visits of a trajectory to a well chosen finite set of domains of phase space. The problem, as far as conservative systems are concerned, is that most orbits are not restricted to this finite set of domains, so that the horseshoe map itself is not conservative. This is the motivation for the develoment of a specially convenient model known as the baker map.

The baker map displays the essential features of classically chaotic motion in such a simplified form as to be almost a caricature $[4,5]$. It may be described as a space-filling horseshoe map that linearizes the hyperbolic motion. Thus, essentially chaotic motion is exhibited, which can be followed through very simple computations. The binary symbolic dynamics propagates vertical strips in the unit square onto horizontal strips. The vertical and horizontal rectangles become narrower, for longer binary codes pertaining to multiple iterations of the baker map. The primary digit specifies either of the two half-squares, so that it is responsible for a coarse-grained description of the motion. The way in which horizontal strips are piled up by the mapping is also determined by the primary binary digit (see Fig. 1).

Higher dimensional generalizations of the baker map have recently been presented [6]. The products of horizontal rectangles for each degree of freedom define hyperparallelepipeds that are stretched and squeezed into horizontal parallelepipeds. Even though the increased dimension admits a much richer range of possibilities for restacking these domains, none of these leads to spiralling motion. Here we show how a rotation can be added which leads to spirals, without violating any of the essential attributes of generalized baker maps.

The various quantization schemes that have been proposed for the baker map generally respect the main division of the square into a pair of vertical rectangles, which split the position states into Hilbert subspaces. These are mapped respectively onto a pair of momentum state subspaces according to the primary binary digit. The discrete and finite nature of the Hilbert space prevents the association of longer codes to ever thinner strips [7]. Thus, to some extent, the primary digit is even more important in quantum mechanics.

Initially, it may have seemed that the introduction of binary symbols to describe evolving quantum systems could only serve as an artificial prop for the study of the semiclassical limit. However, the growing interest in quantum computation has brought the binary structure to the fore. In this context, the quantum baker map has reemerged as an ideal simplified model. Schack and Caves [8] have indeed developed an entire class of quantum baker maps, based on the $2^{N}$-dimensional Hilbert space of $N$ qubits, which include the original quantizations of Balazs and Voros [9] and Saraceno [10] as special cases. The Schack-Caves family was recently further enlarged by Ermann and Saraceno [11], who also explained the general structure of all these maps. We here adopt the Balazs-VorosSaraceno [10] map throughout.

It is important at this stage to distinguish two alternative forms of classical-quantum correspondence for individual baker maps. In the original quantizations $[9,10]$, the classical phase space is two-dimensional, a torus. It is the chaotic motion in this finite region that gives rise to the repulsion of the quantum quasi-energy levels, characteristic of the spectra of random matrices, according to the Bohigas-GiannoniSchmit (BGS) Conjecture [12]. If the quantization is chosen with a value of Planck's constant such that the Hilbert space has exactly $2^{N}$ states, then it is possible to reinterpret the quan- 
tum system to be the tensor product of $N$ two-level systems. This would correspond to a hypercube in a $2 N$-dimensional phase space, but it would be stretching it to ascribe a classical correspondence to this quintessentially quantum system. We shall here keep to the original quantization schemes and, hence, interpret each quantum baker map as corresponding to a classical two-dimensional phase space, so that the coupling of two baker maps corresponds to a four-dimensional phase space. The four possible combinations of primary binary digits, $(0,0),(0,1),(1,0),(1,1)$, then correspond to four squares in the classical position space, $\left(q_{1}, q_{2}\right)$ and hence four parallelepipeds in the unit 4-cube, $\left(q_{1}, q_{2}, p_{1}, p_{2}\right)$ in which the classical motion is defined.

The interaction between baker maps here involves a rotation of the four parallelepipeds into which the primary digits of both maps divide the four dimensional phase space. Choosing this to be of $\pi / 2$, merely exchanges the parallelepipeds, so that the doubled binary decription is respected. The interesting point is that the equilibrium and all the classical periodic orbits become "loxodromic", i.e. the positions spiral outwards, while the momenta spiral inwards, because the eigenvalues of the stability matrix are general complex numbers: Neither are they real, nor do they lie on the unit circle. Obviously, this example models more closely a two dimensional motion for a single particle than any easily conceivable dynamics for a pair of one-dimensional particles. Loxodromic behaviour does not arise for equilibria of simple $p^{2}+V$ Hamiltonians, but it is a generic possibility for the Poincaré map around periodic orbits in higher dimension. So far, not much effort has been made towards quantizing loxodromic motion, except for higher dimensional cat maps [13], another simplified model.

The analysis of the loxodromic baker map is preceeded by a brief review of the ordinary two-dimensional baker map and its quantization. We point out the possibilities for different pilings that lead to the simplest couplings of baker maps [6]. Section 3 then presents the loxodromic baker map and its quantization. It is shown in section 4 that, contrary to the generalized baker maps in [6], the quantum eigenvalue spectrum does not obey a generic random matrix ensemble. The reason for this failure of the BGS conjecture is shown to be that the $\pi / 2$ rotation commutes with the component baker map. This leads to a nontrivial decomposition into a tensor product of two maps, so that the quantum quasi-energy spectrum in this case is highly degenerate.

\section{REVIEW OF CLASSICAL AND QUANTUM BAKER MAPS}

In this section we present the well known classical and quantum ingredients of baker maps. The classical baker transformation is an area preserving, piecewise-linear map, $\mathbf{b}:\left(p_{0}, q_{0}\right) \rightarrow\left(p_{1}, q_{1}\right)$ of the unit square (periodic boundary conditions are assumed) defined as

$$
p_{1}=\frac{1}{2}\left(p_{0}+\varepsilon_{0}\right), \quad q_{1}=2 q_{0}-\varepsilon_{0}
$$

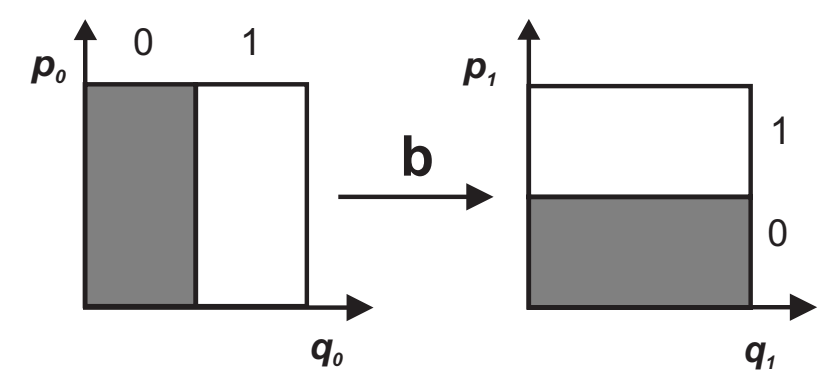

FIG. 1: The baker map propagates a pair of vertical rectangles onto a pair of horizontal rectangles. Inside each separate region the evolution is linear.

where $\varepsilon_{0}=\left[2 q_{0}\right]$, the integer part of $2 q_{0}$. The two regions into which the phase space is separated and their evolution is shown in Fig. 1.

This map is known to be uniformly hyperbolic, the stability exponent for orbits of period $L$ being $L \log 2$. Moreover it admits a useful description in terms of a complete symbolic dynamics. A one to one correspondence between phase space coordinates and binary sequences,

$$
(p, q) \leftrightarrow \ldots \varepsilon_{-2} \varepsilon_{-1} \cdot \varepsilon_{0} \varepsilon_{1} \varepsilon_{2} \ldots, \varepsilon_{i}=0,1
$$

can be constructed in such a way that the action of the map is conjugated to a shift map, i.e., to shifting the dot to the right in the binary sequence above. The symbols are assigned as follows: $\varepsilon_{i}$ is set to zero (one) when the $i$-th iteration of $(p, q)$ falls to the left (right) of the line $q=1 / 2$, i.e. $\left[2 q_{i}\right]=\varepsilon_{i}$. Reciprocally, given an itinerary

$$
\ldots \varepsilon_{-2} \varepsilon_{-1} \cdot \varepsilon_{0} \varepsilon_{1} \varepsilon_{2} \ldots,
$$

the related phase point is obtained through the specially simple binary expansions

$$
q=\sum_{i=0}^{\infty} \frac{\varepsilon_{i}}{2^{i+1}}, \quad p=\sum_{i=1}^{\infty} \frac{\varepsilon_{-i}}{2^{i}} .
$$

Once the dynamics has been mapped to a shift on binary sequences it is very easy to analize the dynamical features of the map. In particular, periodic points are associated to infinite repetitions of finite sequences of symbols.

Due to its piecewise linear nature, the baker map admits a (mixed) generating function which is a piecewise bilinear form,

$$
W_{\varepsilon_{0}}\left(p_{1}, q_{0}\right)=2 p_{1} q_{0}-\varepsilon_{0} p_{1}-\varepsilon_{0} q_{0}, \quad \varepsilon_{0}=0,1 .
$$

It is not defined on the whole space $p_{1}-q_{0}$ but on the classically allowed domains

$$
R_{0}=[0,1 / 2] \otimes[0,1 / 2] \text { and } R_{1}=[1 / 2,1] \otimes[1 / 2,1] .
$$

Though the above generating function will be the starting point for quantization, it must be remembered that it only provides an implicit formula for each iteration of the classical baker map. 
With respect to the quantum map, we will follow the original quantization of Balazs and Voros [9], as later modified by Saraceno [10] to preserve in the quantum map all the symmetries of its classical counterpart. In the mixed representation the baker's propagator can be written as a $D \times D$ block-matrix ( $D$ even):

$$
\left\langle p_{m}\left|\widehat{B}_{D}\right| q_{n}\right\rangle=\left(\begin{array}{cc}
G_{D / 2} & 0 \\
0 & G_{D / 2}
\end{array}\right),
$$

where position and momentum eigenvalues run on a discrete mesh with step $1 / D=h(h=$ Planck's constant $)$, so that

$q_{n}=(n+1 / 2) / D, \quad p_{m}=(m+1 / 2) / D, \quad 0 \leq n, m \leq D-1 ;$

and $G_{D}$ is the antiperiodic Fourier matrix, which transforms from the $q$ to the $p$ basis,

$$
G_{D}=\left\langle p_{m} \mid q_{n}\right\rangle=(1 / \sqrt{D}) e^{-2 \pi i D p_{m} q_{n}} .
$$

It will be useful to consider this matrix as the $q$-representation of the Fourier operator $\widehat{G}_{D}$.

The propagator for the baker map has the standard structure of quantized linear symplectic maps [14],

$$
\left\langle p_{m}\left|\widehat{B}_{D}\right| q_{n}\right\rangle=\left\{\begin{array}{cl}
\sqrt{2 / D} e^{-i 2 \pi D W_{0}\left(p_{m}, q_{n}\right)} & \text { if }\left(p_{m}, q_{n}\right) \in R_{0} \\
\sqrt{2 / D} e^{-i 2 \pi D W_{1}\left(p_{m}, q_{n}\right)} & \text { if }\left(p_{m}, q_{n}\right) \in R_{1} \\
0 & \text { otherwise }
\end{array}\right.
$$

In this quantization, only those transitions are allowed that respect the rule $\left[2 p_{m}\right]=\left[2 q_{n}\right]$, a reflection of the classical shift property. To be able to iterate the quantum baker map, the state must be brought back to the position representation. This is achieved by an inverse Fourier transform, so that the matrix (7) is multiplied by $G_{D}^{-1}$.

Following Schack and Caves [8], we can reinterpret this quantum map as the evolution of $N$ qubits if the dimension of the Hilbert space satisfies $D=2^{N}$. Then the position states can be defined as product states for the qubits in the basis,

$$
\left|q_{n}\right\rangle=\left|\varepsilon_{1}\right\rangle \otimes\left|\varepsilon_{2}\right\rangle \otimes \ldots\left|\varepsilon_{N}\right\rangle,
$$

where $n$ has the binary expansion

$$
n=\varepsilon_{1} \ldots \varepsilon_{n}=\sum_{j=1}^{N} \varepsilon_{j} 2^{N-j}
$$

and $q_{n}=(n+1 / 2) / D=0 \cdot \varepsilon_{1} \ldots \varepsilon_{N} 1$. The connection with the classical baker map is specified by the symbolic dynamics. The bi-infinite strings (3) that determine points in the unit square are made to correspond to sets of orthogonal quantum states. Half of the position states lie in either of the two rectangles, $R_{0}$ or $R_{1}$ defined in (6), which correspond respectively to 0 , or 1 eigenstates of the principal qubit.

The full unitary operator for the quantum baker can be written explicitly as

$$
\widehat{B}_{D}=\widehat{G}_{D}^{-1}\left[\hat{1}_{2} \otimes \widehat{G}_{D / 2}\right]
$$

where $\hat{1}_{2}$ is the unit operator for the first bit and $\widehat{G}_{D / 2}$ is the Fourier operator on the remaining qubits. The operator in the

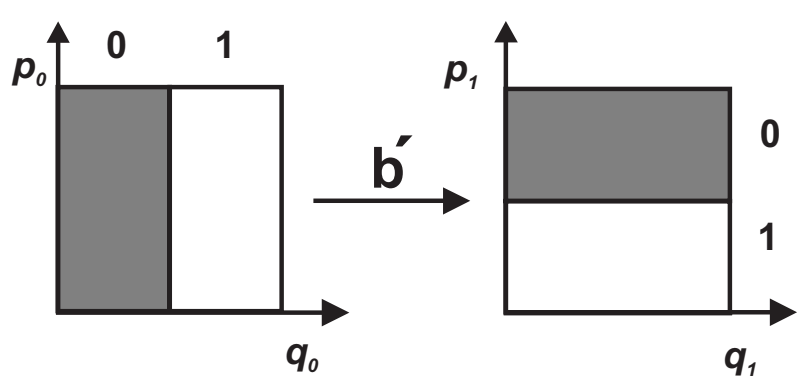

FIG. 2: The alternative baker map also propagates a pair of vertical rectangles onto a pair of horizontal rectangles, but their stacking is interchanged.

square brackets preserves the first qubit, while evolving separately the remaining qubits, within each domain $R_{\varepsilon_{1}}$. It is the final inverse Fourier operator that mixes the principal qubit in with the rest, because it acts globaly on the states in both domains. This step is not explicitated in the mixed representation (7).

So far we have only allowed for a single possibility in which to stack the rectangles in the baker transformation, but alternative to (1), the classical map, $\mathbf{b}^{\prime}:\left(p_{0}, q_{0}\right) \rightarrow\left(p_{1}, q_{1}\right)$, shown in Fig. 2,

$$
p_{1}=\frac{1}{2}\left(p_{0}+1-\varepsilon_{0}\right), \quad q_{1}=2 q_{0}-\varepsilon_{0},
$$

has very similar properties. This variation is tantamount to reversing the primary classical bit and it corresponds to the quantum map $\widehat{B}^{\prime}$, represented by the matrix:

$$
\left\langle p_{m}\left|\widehat{B}_{D}^{\prime}\right| q_{n}\right\rangle=\left(\begin{array}{cc}
0 & G_{D / 2} \\
G_{D / 2} & 0
\end{array}\right) .
$$

In other words, we here substitute the operator $\hat{I}_{2}$, which acted on the principal qubit, by $\widehat{X}_{2}$ represented by the Pauli matrix:

$$
X_{2}=\left(\begin{array}{ll}
0 & 1 \\
1 & 0
\end{array}\right)
$$

so that $\widehat{B}_{D}^{\prime}=\widehat{G}_{D}^{-1}\left[\widehat{X}_{2} \otimes \widehat{G}_{D / 2}\right]$.

Of course, we are free to substitute any other unitary operator acting on the primary qubit, but it is only $\widehat{B}^{\prime}$ that can be interpreted classically as an equivalent alternative piling of the baker map. We can still split up the evolution into domains equivalent to (6), though with a different matching of $q_{0}$ and $p_{1}$ segments. The classical evolution within each domain is again determined by classical generating functions like (5) which becomes the exponent of the propagator.

If we allow a finite probability for a stacking fault in the classical baker map, the evolution acquires a random component. However, if the piling order depends on the coarsegrained position of another baker map, the overall motion will again be purely deterministic. Being that each baker map is thoroughly chaotic, it will be hard to distinguish the random motion of one of the components taken on its own from a truly stochastic system. In [6] we allowed the baker to choose between these alternatives, depending on its interaction with another baker. Though this led to surprisingly rich structures, 


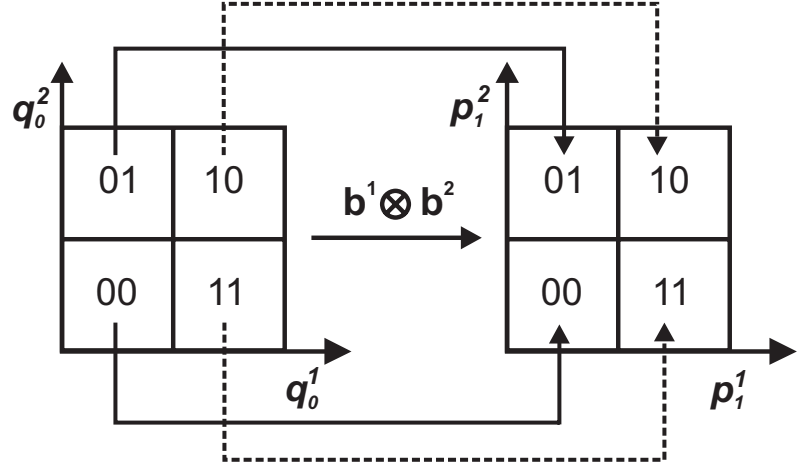

FIG. 3: The partition of phase space into disjoint domains is respected by the product of baker maps. 4D-parallelepipeds, projecting onto squares in the position space are transformed onto horizontal parallelepipeds that project onto squares in momentum space.

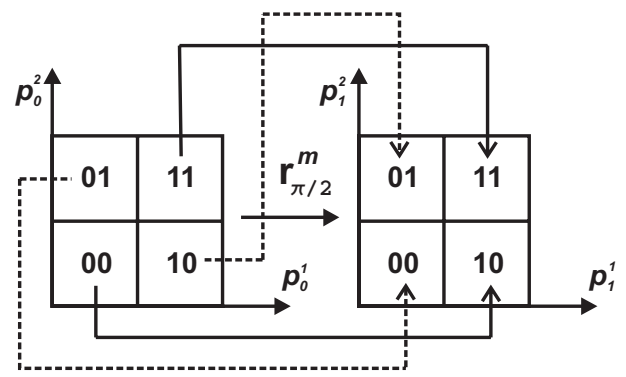

FIG. 4: Possible repiling of the partition of the domains of the product of baker maps.

no spiraling motion could be generated in the corresponding classical map. This possibility is now realized by rotating the domains into which the baker's phase space is partitioned.

\section{DEFINITION OF THE LOXODROMIC BAKER MAP}

The direct product of a pair of classical baker maps, $\mathbf{b}^{1} \otimes$ $\mathbf{b}^{2}$, is itself a generalized baker map defined within a 4Dhypercube, $\left(p^{1}, q^{1}\right) \otimes\left(p^{2}, q^{2}\right)$. Indeed the motion is hyperbolic and the partition of phase space into disjoint domains is again respected. These are specified by a doubled binary code, $\varepsilon_{0}=\varepsilon_{0}{ }^{1} \otimes \varepsilon_{0}{ }^{2}$, defining vertical 4D-parallelepipeds that project onto squares in the position space $\left(q^{1}, q^{2}\right)$, while covering the full available momentum space. The effect of a single iteration of this product map is to transform these domains onto horizontal parallelepipeds that project onto squares in momentum space, as shown in Fig. 3.

The main difference between this product map and the generalized bakers defined in [6] is that in the latter case the symbolic mapping is no longer the identity. One could certainly define a generalized baker by repiling the product maps within each domain so as to obtain the symbolic evolution displayed in Fig. 4, though this possibility was not singled out in [6].

However, the same effect (as far as the symbolic evolution is concerned) can be obtained by combining the product baker with a canonical point transformation, $\mathbf{r}_{\pi / 2}^{\mathbf{m}}$, that simply rotates
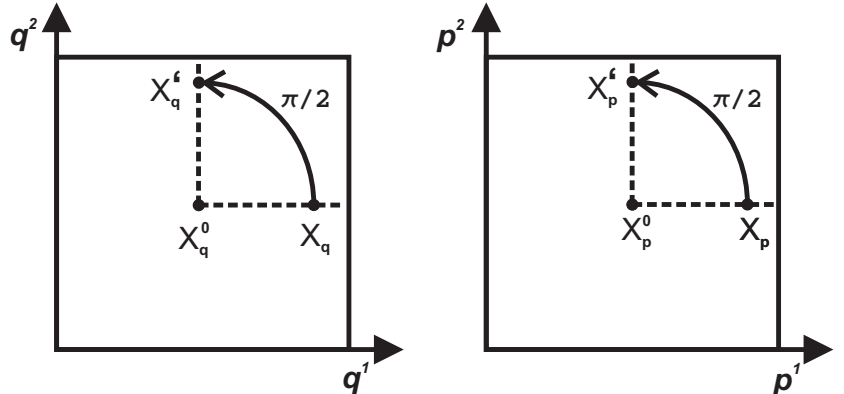

FIG. 5: A canonical point transformation, $\mathbf{r}_{\pi / 2}^{\mathbf{m}}$, that simply rotates both the position and the momentum spaces around the midpoint of the hypercube, $\mathbf{m}=(1 / 2,1 / 2,1 / 2,1 / 2)$, has the same effect on the partition of the product of baker maps as the repiling in Fig. 4.

both the position and the momentum spaces around the midpoint of the hypercube, $\mathbf{m}=(1 / 2,1 / 2,1 / 2,1 / 2)$, as shown in Fig. 5.

This loxodromic baker map, $\mathbf{r}_{\pi / 2}^{\mathbf{m}} \circ\left[\mathbf{b}^{1} \otimes \mathbf{b}^{2}\right]=\mathbf{b}_{\mathbf{L}}$ : $\left(p_{0}, q_{0}\right) \rightarrow\left(p_{1}, q_{1}\right)$ is given explicitely by

$$
\begin{aligned}
& p_{1}{ }^{1}=1-\frac{1}{2} p_{0}{ }^{2}-\frac{1}{2} \varepsilon_{0}{ }^{2} \\
& p_{1}{ }^{2}=\frac{1}{2} p_{0}{ }^{1}-\frac{1}{2} \varepsilon_{0}{ }^{1} \\
& q_{1}{ }^{1}=1-2 q_{0}{ }^{2}-\frac{1}{2} \varepsilon_{0}{ }^{2} \\
& q_{1}{ }^{2}=2 q_{0}{ }^{1}-\frac{1}{2} \varepsilon_{0}{ }^{1} .
\end{aligned}
$$

This is no longer a product map within each separate domain and Fig. 6 shows how an orbit spirals onto a fixed point in momentum space and spirals outwards in position space.

The loxodromic baker map can also be described by symbolic dynamics. This now has a four letter alphabet, two binary digits, instead of one for the ordinary baker map. Furthermore, the relation between the symbolic description and the phase space trajectories is not so direct. The details may be obtained in [16].

The quantum version of the loxodromic baker map is defined in strict correspondence with the foregoing classical construction. This is always possible for linear classical evolutions, i.e. we can define a quantum unitary transformation, $\widehat{R}_{\pi / 2}^{\mathbf{m}}$, which corresponds to the classical transformation, $\mathbf{r}_{\pi / 2}^{\mathbf{m}}$, so that, in combination with the definition of the usual baker map (13), we then stipulate that

$$
\widehat{B}_{L}=\widehat{R}_{\pi / 2}^{\mathbf{m}} \widehat{B}_{D} \otimes \widehat{B}_{D}
$$

The only thing that is now missing is the full definition of the rotation operator in the above formula. Recalling that the classical rotation is about the midpoint $\mathbf{m}$ in phase space, it follows that, if the rotation operator about the origin by an angle $\alpha$ is

$$
\widehat{R}_{\alpha}^{0}=\exp \left[-i \alpha\left(\widehat{p}_{2} \widehat{q}_{1}-\widehat{q}_{2} \widehat{p}_{1}\right)\right]
$$



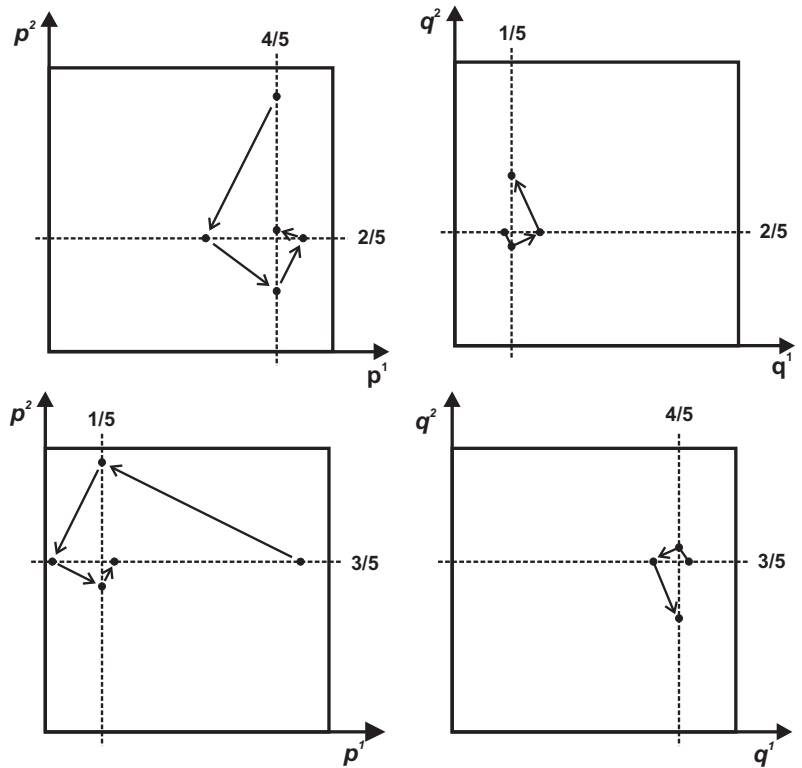

FIG. 6: Typical classical trajectories of the loxodromic baker map spiral out of the fixed points in the q-plane and into the fixed points in the p-plane. Every four iterations they return to the same radial line, because the rotation angle is $\pi / 2$.

then

$$
\widehat{R}_{\alpha}^{\mathbf{m}}=\widehat{T}_{\mathbf{m}} \widehat{R}_{\alpha}^{0} \widehat{T}_{-\mathbf{m}}
$$

where $\widehat{T}_{\xi}$ is the quantum operator corresponding to a uniform translation (or displacement) by the phase space vector $\xi$ (see e.g.[18]).

A considerable simplification is possible for the $\pi / 2$ rotation, which is the only one allowed for a generalized baker map. In this case, it is easy to verify that the classical rotation about the midpoint, $\mathbf{m}$, can be written as a composition

$$
\mathbf{r}_{\pi / 2}^{\mathbf{m}}=\mathbf{t}_{\eta} \circ \mathbf{r}_{\pi / 2}^{0}
$$

where $\mathbf{t}_{\eta}$ is the classical translation by the vector $\eta=$ $(1,0,1,0)$ and $\mathbf{r}_{\pi / 2}^{0}$ the $\pi / 2$ rotation around the origin. Therefore, we can quantize this form directly so as to obtain

$$
\widehat{R}_{\pi / 2}^{\mathbf{m}}=\widehat{T}_{\eta} \widehat{R}_{\pi / 2}^{0}
$$

\section{SYMMETRIES AND LEVEL REPULSION}

The celebrated BGS conjecture predicts the correspondence between chaotic classical motion and quantum level repulsion. Specifically, for quantum maps, the eigenvalue spectrum of the unitary operator should have fluctuations in agreement with the canonical CUE, or COE ensembles of random matrix theory (RMT). Exceptions do arise because of symmetries, or even more subtle arithmetic anomalies which may be hidden in the definition of the map. However, the BGS conjecture has become so well established that its negation is taken as a sure sign that hidden structure should be sought out.

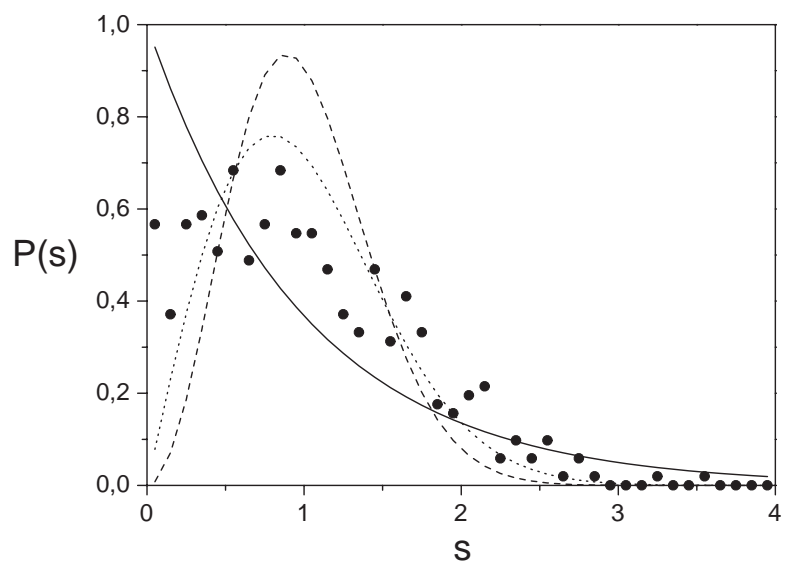

FIG. 7: Distribution of level spacings -normalized to unit averagefor the repiled baker maps (full dimension equal to 512; big dots). We also display the predictions of random matrix theory (dotted line: COE, dashed: CUE) and the Poisson distribution (full line).

In relation to the baker map, it should be noted that the original quantization by Balazs and Voros [9] did not conform properly to the conjecture. Saraceno then discovered that this form of quantization did not incorporate an exact classical symmetry and proposed a modification [10]. The individual symmetry classes of this new map are satisfactory examples of the BGS conjecture as can be verified in [15].

Even though the direct product of two baker maps is certainly a chaotic classical system, its quantization does not satisfy the BGS conjecture. Indeed, the eigenvalues will be the products of the baker eigenvalues, leading to degeneracies instead of level repulsion. In general, the addition of some interaction between the two maps, while taking the precaution to preserve the hyperbolicity of the classical motion, should lead again to an RMT spectrum. In the case of the generalized "repiled" baker maps studied in [6] the level spacing distribution, shown in Fig. 7, does not comply with the BGS conjecture. Its intermediate nature between a Poisson spectrum and a COE spectrum suggests the nearness of a symmetry, as was found for the original Balasz-Voros quantization of the baker map. However, Fig. 8 clearly rules this possibility out in the case of the loxodromic baker map. The Poisson profile indicates independent samples and, furthermore, there are many degeneracies, just as with the simple product map. Therefore we must seek out the symmetry that is responsible. It turns out that this involves the reflection symmetry of the classical baker map, $\mathbf{R}:(q, p) \rightarrow R(q, p)=(1-q, 1-p)$, included in the Saraceno quantization [10]. Of course, both factors of the product map, $\mathbf{b}^{1} \otimes \mathbf{b}^{2}$, commute with the respective reflections, $\mathbf{R}_{1}$ and $\mathbf{R}_{2}$. Another obvious symmetry of the product map is the permutation, $\mathbf{P}:\left(q_{1}, p_{1}, q_{2}, p_{2}\right) \rightarrow$ $P\left(q_{1}, p_{1}, q_{2}, p_{2}\right)=\left(q_{2}, p_{2}, q_{1}, p_{1}\right)$. Combining these, we then obtain:

$$
\begin{gathered}
\mathbf{R}_{1} P\left(q_{1}, p_{1}, q_{2}, p_{2}\right)=R_{1}\left(q_{2}, p_{2}, q_{1}, p_{1}\right)= \\
\left(1-q_{2}, 1-p_{2}, q_{1}, p_{1}\right)=\mathbf{r}_{\pi / 2}\left(q_{1}, p_{1}, q_{2}, p_{2}\right) .
\end{gathered}
$$




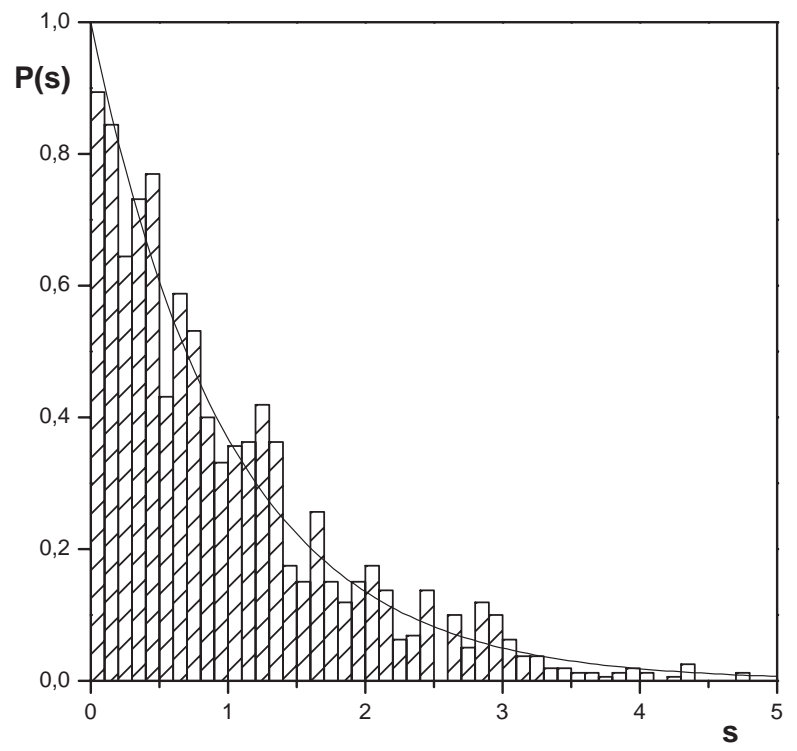

FIG. 8: Histogram of level spacings for eigenangles of a loxodromic baker map with dimension equal to 1296 . The smooth curve corresponds to the Poisson spectrum.

Thus, we find that the special nature of the $\pi / 2$ rotation, that was chosen because it respects the geometrical divisions of the baker map, can now be reinterpreted as a particular product of the symmetries of $\mathbf{b}^{1} \otimes \mathbf{b}^{2}$.

It is then neither surprising, nor hard to prove, that $\mathbf{r}_{\pi / 2}$ itself is a symmetry of the classical and the quantum product baker map. The consequence is that $\widehat{R}_{\pi / 2}$ and $\widehat{B}_{D} \otimes \widehat{B}_{D}$ have common eigenfunctions and they must share these with $\widehat{B}_{L}$, as defined by (21). Thus, if we divide the spectrum of the product map into the symmetry classes of the quantum rotation $\widehat{R}_{\pi / 2}$, with eigenvalues $\{+1,-1,+i,-i\}$, the eigenvalues of $\widehat{B}_{L}$ in each class are just the corresponding multiple of the eigenvalues of the quantized product map. In conclusion, the loxodromic baker map belongs to the class of quantized chaotic maps which fail to satisfy the BGS conjecture because of a symmetry.

\section{DISCUSSION}

The quantization of spiraling chaotic motion has not received the attention it deserves, because, so far, work has concentrated on two-dimensional maps. For systems with many degrees of freedom, it must be borne in mind that Poincaré maps will generally exhibit spiraling behaviour along with the more familiar features studied in two-dimensional maps. The complexity of higher dimensional motion encourages the search for specially simple models with which to study the classical-quantum correspondence and the semiclassical limit. The generalized baker map presented here was an obvious candidate, but the restriction to rotations of $\pi / 2$, amounting roughly to a permutation of the variables between the two coupled maps, turns out to be too severe. The commutation of this rotation with the baker product map leads to a totally unsatisfactory eigenvalue spectrum for a chaotic quantum map. This is in sharp contrast to even simpler couplings developed for baker maps in [6]

Another possibility that has also been investigated are loxodromic cat maps, i.e. linear maps on the 4-torus [13]. There is then a richer range of possibilities, but still there are restrictions to the distribution of eigenvalues common to all cat maps. These arise from the necessity of quantum cat maps to be periodic, albeit the period may be very high. Presumably, the search for simple loxodromic quantum models will only heat up when a loxodromic algorithm is invented for quantum information theory.
[1] V. I. Arnold, Mathematical Methods of Classical Mechanics (Springer, New York, 1978).

[2] J. Guckenheimer and P. Holmes, Nonlinear Oscillations, Dynamical systems and Bifurcations of Vector Fields (Springer, New York, 1983).

[3] A. M. Ozorio de Almeida, Hamiltonian Systems: Chaos and Quantization (Cambridge University Press, Cambridge, 1988).

[4] V. I. Arnold and A. Avez, Ergodic Problems of Classical Mechanics (Benjamin, New York, 1968).

[5] A. J. Lichtenberg and M. A. Lieberman, Regular and Stochastic Motion (Springer, New York, 1983).

[6] R. O. Vallejos, P. R. del Santoro, and A. M. Ozorio de Almeida, J. Phys. A: Math. Gen. 39, 5163 (2006).

[7] M. Saraceno and A. Voros, Physica D 79, 206 (1994).

[8] R. Schack and C. M. Caves, Appl. Algebra Eng. Commun. Comput. 10, 305 (2000).
[9] N. L. Balazs and A. Voros, Ann. Phys. 190, 1 (1989).

[10] M. Saraceno, Ann. Phys. 199, 37 (1990).

[11] L. Ermann and M. Saraceno, arXiv:nlin.CD/060602.

[12] O. Bohigas, in Chaos and Quantum Physics -Proc. Les Houches Summer School 1989-, ed M. J. Giannoni, A. Voros and J. Zinn-Justin (North-Holland, Amsterdam, 1991).

[13] A. M. F. Rivas, M. Saraceno, and A. M. Ozorio de Almeida, Nonlinearity 13, 341 (2000).

[14] A. M. Ozorio de Almeida and M. Saraceno, Ann. Phys. 210, 1 (1991).

[15] P. W. O'Connor and S. Tomsovic, Ann. Phys. 207, 218 (1991).

[16] P. R. del Santoro, PhD Thesis (in preparation).

[17] J. H. Hannay, J. P. Keating, and A. M. Ozorio de Almeida, Nonlinearity 7, 1327 (1994).

[18] A. M. Ozorio de Almeida, Phys. Rep. 295, 265 (1998). 